\title{
Chaotic boundaries of nematic polymers in mixed shear and extensional flows
}

\author{
M. Gregory Forest, Ruhai Zhou \\ Department of Mathematics \\ University of North Carolina at Chapel Hill \\ Chapel Hill, NC 27599 \\ Qi Wang \\ Department of Mathematical Sciences \\ Florida State University \\ Tallahassee, FL 32306
}

April 6, 2004 


\begin{abstract}
Chaotic orientational dynamics of sheared nematic polymers is documented in laboratory experiments on model systems [1]; a question arises as to how robust the phenomenon is with respect to composition and flow properties. The Doi-Hess kinetic theory for infinitely thin rods predicts chaotic monodomain dynamics $[2,3,4]$ over a range of concentrations $(c)$ at which the quiescent phase is ordered, and within a window of shear rates $(\dot{\gamma})$ for each concentration. Thus, a bounded region of $(c, \dot{\gamma})$ has chaotic attractors [5]. Our goal here is to relax two idealizations of these numerical studies, and to address the issue of robustness. Pure shear is modified by a planar straining flow of arbitrary strength, and the macromolecules are endowed with arbitrary aspect ratio. We then predict the deformation of the chaotic parameter region in [5] due to these 2 physical effects, applying a correspondence principle developed in [6] together with the numerical bifurcation software AUTO. A typical question addressed is: how strong of an extensional flow component is necessary to arrest chaotic behavior in pure shear of a nematic liquid? In answering this question, an intriguing prediction emerges: a finite-strength straining component can induce chaotic orientational dynamics. Indeed, all known sheared oscillatory attractors (tumbling, wagging, and kayaking) exist at shear rates below the onset of chaos and may be driven through transitions to chaos by adding a straining flow component of prescribed extension rate.
\end{abstract}


Nematic polymers may exhibit chaotic phenomena in shear-dominated flows in the laboratory (see, e.g., [1]) and in models [2, 3, 4]. Using the kinetic theory of Doi and Hess, for infinitely thin rods or platelets in pure shear, the authors have produced a phase diagram in [5] of all stable states and phase transitions in different regions of the dimensionless parameter space $(N, P e)$. Here $N$ denotes the intensity of the nematic excluded-volume potential (a dimensionless polymer concentration $c$ ), and the Peclet number $P e=\dot{\gamma} / D r$ is the shear rate $(\dot{\gamma})$ normalized by molecular rotational relaxation rate $(D r)$. For the Maier-Saupe potential and a constant rotational diffusion rate, the chaotic region lies within the rectangle $4.7<N<5.4,1<P e<4.5$. If we model orientation-dependent rotational diffusion, the chaotic regime persists, with slightly modified bounds, but without qualitative changes in the phase diagram [5]. Our goal here is to track the boundaries of the chaotic region due to two related and unavoidable physical effects: finite molecule aspect ratio and the addition of straining flow in the plane of shear. These boundaries inform the robustness of shearinduced rheochaos of nematic polymers: how anisotropic must the molecules be, and what extensional flow strength is required for the chaotic response to persist or be arrested?

We now briefly review the kinetic theory. Let $f(\mathbf{m}, t)$ be the orientational probability distribution function (PDF) for rod-like, rigid, extremely high-aspect-ratio spheroidal molecules with axis of symmetry $\mathbf{m}$ on the unit sphere $S^{2}$. The Smoluchowski equation for $f(\mathbf{m}, t)$ in a flow field $\mathbf{v}$ is given by Doi [7], Hess [8]. We assume constant rotational diffusivity (Dr is constant) in this study. The Smoluchowski equation, made dimensionless through this rotational diffusivity constant, takes the form

$$
\begin{aligned}
& \frac{\partial f}{\partial t}=\mathcal{R} \cdot\left(\mathcal{R} f+\frac{1}{k T} f \mathcal{R} V\right)-\mathcal{R} \cdot[\mathbf{m} \times \dot{\mathbf{m}} f] \\
& \dot{\mathbf{m}}=\mathbf{\Omega} \cdot \mathbf{m}+a[\mathbf{D} \cdot \mathbf{m}-\mathbf{D}: \mathbf{m m m}]
\end{aligned}
$$

where, $\mathcal{R}=\mathbf{m} \times \partial / \partial \mathbf{m}$ is the rotational gradient operator, and $V$ is the Maier-Saupe potential

$$
V=-\frac{3}{2} N k T \mathbf{m m}:\langle\mathbf{m m}\rangle
$$


The dimensionless flow field for pure shear is

$$
\mathbf{v}=\operatorname{Pe}(y, 0,0)
$$

$\mathbf{D}$ and $\boldsymbol{\Omega}$ in (1) are the corresponding rate-of-strain and vorticity tensors, which are the symmetric and anti-symmetric part of $\nabla \mathbf{v}$, respectively. So $\mathbf{v}=(\boldsymbol{\Omega}+\mathbf{D}) \cdot \mathbf{x}$, where $\mathbf{x}=$ $(x, y, z)$ are Cartesian coordinates with $x$ the flow direction, $y$ the flow-gradient direction, and $z$ the vorticity axis; $k T$ in equation (1) is the Boltzmann factor. The aspect ratio parameter $a$ is defined by

$$
a=\frac{r^{2}-1}{r^{2}+1}
$$

where $r$ is the ratio of the rod length to the diameter. Figure 1 shows the relationship between $r$ and $a$. For very long rods, $r \rightarrow \infty, a \rightarrow 1$; for very thin disks, $r \rightarrow 0, a \rightarrow-1$.

In [9], the bifurcation diagram is given for $|a|=1$, i.e., $r=\infty$ or 0 . The chaotic parameter region is blown up in Figure 2. We first characterize the persistence of the chaotic region for physical aspect ratio parameters, $|a|<1$. Intuitively, we expect the chaotic region to shrink as the aspect ratio drops. Indeed, lowering $|a|$ is equivalent to adding a straining component to the flow and maintaining the aspect ratio $r=\infty$, a "trade-off" that has been noted by several authors, and precisely formulated in terms of the solution space of kinetic and mesoscopic models in $[6,10]$. Later, we will use this fact to characterize the extensional flow perturbation strength necessary to tame the chaotic regime (and surprisingly, to possibly grow the chaotic regime when pure shear rates are not strong enough).

We amplify two vertical slices in Figure 2 corresponding to fixed nematic concentrations, $N=5$ and $N=5.2$, and explore variability with respect to $|a(r)|<1$. We first write the PDF as a high-order truncated spherical harmonic expansion, and derive a system of ordinary differential equations for each harmonic amplitude [10, 11, 12]. Then we employ the continuation software AUTO [13] to identify stable attractors and bifurcation diagrams. Finally we use a 4th order spectral-deferred-correction time-integration method to investigate 
properties of attracting limit cycles and chaotic orbits. These results determine the attractor taxonomy listed in the caption of Figure 2; see [4, 5, 9] for details.

For $N=5.2$, the bifurcation diagram versus $P e$ that underlies the phase diagram of Figure 2 is detailed in Figure 3a. From these stable and unstable branches and bifurcations, we employ numerical continuation versus $a$ for fixed $N=5.2$. The set of all stable states in the parameter rectangle $0.8<a<1.13,1<P e<6$ is extracted from this 2-parameter study, then compiled in Figure 3b. There are 9 separate stability regions; in each region all stable state(s) are given. The boundaries of each region are phase transitions of the flowing nematic liquid, characterized from AUTO in terms of bifurcations. The chaotic region is confined within the rectangle $0.98<a<1.12,1.6<P e<4.2$. We note that the boundary of the chaotic region is associated with a periodic doubling cascade $[2,4,5]$.

From Figure 3b, for physical aspect ratio parameters $1<r<\infty(0<a<1)$, the shear rate interval of Pe with chaotic attractors contracts as $r$ decreases. For pure shear flow and $N=5.2$, the critical value at which chaotic response terminates is $\left|a^{*}\right| \approx 0.98$, which corresponds to platelets of aspect ratio $r \approx 10^{-1}$, and rods of aspect ratio $r \approx 10$. For nematic polymers of less extreme aspect ratios, $1 / 10<r<10$, the chaotic range of $P e$ has vanished at this concentration. The corresponding results of Figure $3 \mathrm{~b}$ for the $N=5$ slice of Figure 2 is given in Figure 4. Note that we have scaled the axes $a$ and $P e$, yet only slightly, and the $N=5,5.2$ phase diagrams are remarkably similar.

We emphasize that our methods equally characterize the boundaries in parameter space of other attractors, both steady (FA, OS) and unsteady ( $\mathbf{T}, \mathbf{W}, \mathbf{K}_{1}, \mathbf{K}_{2}$ ), and also map out regions of unique versus bi- and tri-stability. Note further that we have allowed $a$ to wander outside the apparently physical bounds $|a|<1$, which now will be physically connected in terms of a straining flow perturbation of shear.

The next physical effect to explore is the admission of an arbitrary extensional flow component in the previously pure shear velocity field. That is, the dimensionless gradient is 
now allowed the following form

$$
\nabla \mathbf{v}=\left(\begin{array}{ccc}
p_{1} & P e & 0 \\
0 & -p_{1} & 0 \\
0 & 0 & 0
\end{array}\right)
$$

where the non-dimensional parameter $p_{1}=\dot{\epsilon} / D r$ is the extensional flow rate $(\dot{\epsilon})$ normalized by rotational diffusion rate $(D r)$. Note the flow parameter ratio

$$
\chi=\frac{p_{1}}{P e}=\frac{\dot{\epsilon}}{\dot{\gamma}}
$$

is the ratio of extension rate to shear rate.

In [6], we formulated a correspondence principle between nematic polymers in general planar linear flow and pure shear flow. From that principle, the monodomain response of nematic polymers with aspect ratio parameter $a$ in the flow field (5) is identical to the monodomain response of another nematic polymer liquid with aspect ratio parameter $\bar{a}$ in pure shear flow with shear rate $P e$, where the molecular aspect ratios are related by

$$
\bar{a}=a \cdot \sqrt{1+4\left(\frac{p_{1}}{P e}\right)^{2}} .
$$

As amplified in [6], $\bar{a}$ need not correspond to a physical molecular aspect ratio; that is, $|\bar{a}|>1$ is allowable in pure shear and simply corresponds to an extensional flow component of a "real liquid" $(|a|<1)$ in addition to the shear component Pe.

We now revisit Figure $3 \mathrm{~b}$ in this light, and observe that the phase diagram for $a \geq 1$ is equivalent to fixing $a=1$, renaming the axis $\bar{a}$ in Figure $3 \mathrm{~b}$, and admitting a flow field continuation from $p_{1}=0$ to $p_{1}=\frac{P e}{2} \sqrt{\bar{a}^{2}-1}$ (an equivalent statement of $(7)$ with $a=1$ ).

These arguments allow us to transform Figure 3b for $a \geq 1$ into another phase diagram with direct physical relevance. For fixed concentration $N=5.2$ and extreme aspect ratio $(a=1)$, Figure 5a gives the phase diagram of attractors and phase transitions versus shear rate $P e$ and the relative strength $(\chi)$ of extension rate to shear rate

$$
\chi=\frac{p_{1}}{P e}=\frac{1}{2} \sqrt{\left(\frac{\bar{a}}{a}\right)^{2}-1} .
$$


(In fact, for any physical aspect ratio, $|a|<1$, we can give a similar phase diagram using the formula (8).) Note the horizontal axis of Figure 5a coincides with the $a=1$ vertical slice of Figure $3 \mathrm{~b}$ and the bifurcation diagram Figure 3a.

Observe from Figure 2 and Figure 3, and the horizontal axis of Figure 5a, for pure shear flow $\chi=0$, the $P e$ window of chaos is $2.69<P e<4.1$. As we amplify a small extensional flow component, $\chi>0$, the chaotic window of $P e$ grows! The right boundary increases until $\chi \approx 0.1$, then sharply drops as $\chi$ approaches 0.25 to hit the left boundary. The left boundary also shows that, when the extension rate $(\dot{\epsilon})$ approaches a critical value, chaotic dynamics is created from the otherwise periodic monodomain dynamics in pure shear. From the phase diagrams of Figure 5 for either concentration, one can consider a sequence of vertical slices, e.g., $P e=2.0,2.4,2.6$ of Figure 5a. These slices correspond to the transition sequence of attractors versus $\chi=\dot{\epsilon} / \dot{\gamma}$, starting from pure shear, $\chi=0$. We find that, $\mathbf{T}, \mathbf{W}, \mathbf{K}_{1}$, and $\mathbf{K}_{2}$ attractors all exist for sufficiently weak extension rates, then undergo a transition to chaos as $\chi$ increases! Furthermore, the chaotic attractors persist for a finite interval of $\chi$ that varies with $P e$ and $N$, as detailed in Figure 5a, 5b.

The phase diagram for $N=5.2$ (Figure 5a) also shows that with $|a|=1$, for sufficiently strong extension rate, $\chi>0.25$, chaotic response has been completely arrested, independent of shear rate $(P e)$. The top boundary of the chaotic region $(\mathbf{C H})$ in Figure 5a,5b conveys the answer to the question posed in the abstract for two concentrations of extreme aspect ratio nematic liquids. The left boundary of the chaotic region in shear rate versus extension rate $(P e, \chi)$, because the boundary has negative slope, conveys the intriguing prediction that a regular periodic regime of sheared nematic polymers can be driven chaotic by introducing a pure extensional flow perturbation!

Finally we superimpose the chaotic regions for $N=5$ and $N=5.2$ on the same $(P e, \chi)$ scales in Figure 6. This comparison shows how the chaotic flow domain shifts versus concentration, yet remains qualitatively similar. Clearly, one can use these tools to calculate similar phase diagrams for any concentration $N$ and aspect ratio parameter $a$. 
Conclusion: The parameter regime of chaotic orientational dynamics of shear-dominated flowing nematic polymers has been explored with a combination of numerical continuation software and a correspondence principle of the Doi-Hess kinetic theory. The dynamics and phase transitions have been characterized versus molecular composition (concentration and aspect ratio), flow type and flow rate for a linear combination of planar shear and extension. The chaotic boundaries are mapped out for selected 2-dimensional parameter regimes. The chaotic orbits identified previously for infinite aspect ratio rods $[2,5]$ are shown to persist to finite aspect ratios on the order of 10. An intriguing prediction emerges from our numerical phase diagram: the addition of an extensional flow component at sub-critical shear rates can resonate chaotic flow response in an otherwise periodic attractor, including tumbling, wagging, and out-of-plane kayaking limit cycles. On the other hand, any given chaotic response for a fixed parameter set can be driven into a regular, flow-aligned orientational distribution by addition of a finite straining component to the flow. Taken together, these results indicate a degree of robustness as well as intrigue in phase flows of nematic polymers.

\section{Acknowledgment}

Effort sponsored by the Air Force Office of Scientific Research, Air Force Materials Command, USAF, under grant number F49620-02-1-0086, and the National Science Foundation through grants DMI-0115445, DMS-0204243, DMS-0308019. This work is supported in part by the NASA University Research, Engineering and Technology Institute on Bio Inspired Materials (BIMat) under award No. NCC-1-02037.

\section{References}

[1] R. Bandyopadhyay, G. Basappa, A.K. Sood, Phys. Rev. Lett. 84(9), 2022-2025 (2000).

[2] M. Grosso, R. Keunings, S. Crescitelli, P.L. Maffettone, Phys. Rev. Lett. 86(14), 31843187 (2001). 
[3] G. Rienacker, M. Kroger, S. Hess, Phys. Rev. E 66(4), 040702 (2002).

[4] M.G. Forest, Q. Wang, Rheol. Acta, 42, 20-46 (2003).

[5] M.G. Forest, Q. Wang, R. Zhou, submitted to Rheol. Acta (2003).

[6] M.G. Forest, Q. Wang, R. Zhou, E.P. Choate, J. Non-Newt. Fluid Mech., in press (2004).

[7] M. Doi, J. Polym. Sci., Polym. Phys. Ed. 19, 229-243 (1981).

[8] S. Hess, Z. Naturforsch Teil 31A, 1034 (1976).

[9] M.G. Forest, Q. Wang, R. Zhou, Rheol. Acta, 43(1), 17-37 (2004).

[10] M.G. Forest, R. Zhou, Q. Wang, Phys. Rev. E, 66(3), 031712 (2002).

[11] R.G. Larson, H. Ottinger, Macromolecules 24, 6270-6282 (1991).

[12] V. Faraoni, M. Grosso, S. Crescitelli, P.L. Maffettone, J. Rheol. 43, 829-843 (1999).

[13] E.J. Doedel, et al., AUTO97: Continuation and bifurcation software for ordinary differential equations, Concordia University (1997). 


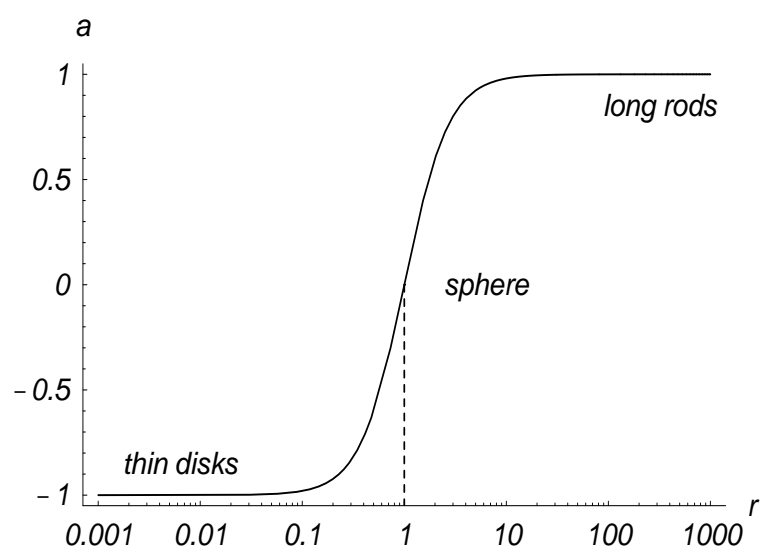

Figure 1: The shape parameter $a$ versus molecule aspect ratio $r$. 


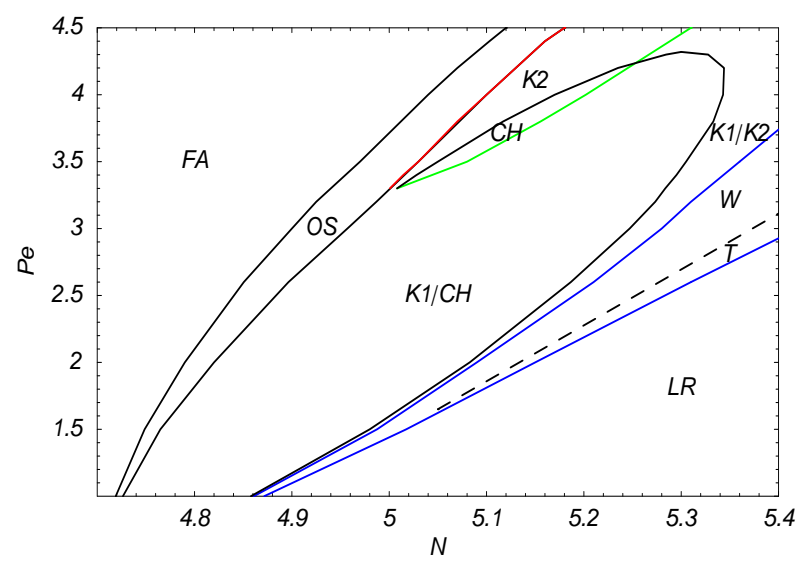

Figure 2: (Reproduced with permission of the publishers from [5].) Bifurcation diagram of all stable states of the Doi-Hess kinetic theory for the parameter domain $4.7<N<5.4$, $1<P e<4.5$ with constant rotational diffusivity constant and infinite aspect ratio $r=\infty$. Notation: FA denotes flow-aligned steady states; OS denotes out-of-plane steady states which always occur in symmetric pairs; K1 is the Larson-Ottinger kayaking limit circle in which the peak orientation of the PDF rotates about the vorticity axis; K2 is a kayaking state in which the peak orientation rotates about a tilted direction (these states also occur in symmetric pairs); LR is the logrolling steady state with peak orientation along the vorticity axis; $\mathbf{T}$ is the tumbling limit circle in which the peak orientation continues to rotate in the deformation plane; $\mathbf{W}$ is the wagging state in which the peak orientation is also in the deformation plane but oscillates around the flow direction; and $\mathbf{C H}$ denotes chaotic dynamics. The Pe dependent bifurcation diagram for fixed concentration $N=5.2$ (a vertical slice of this figure) is given in Figure 3a. 

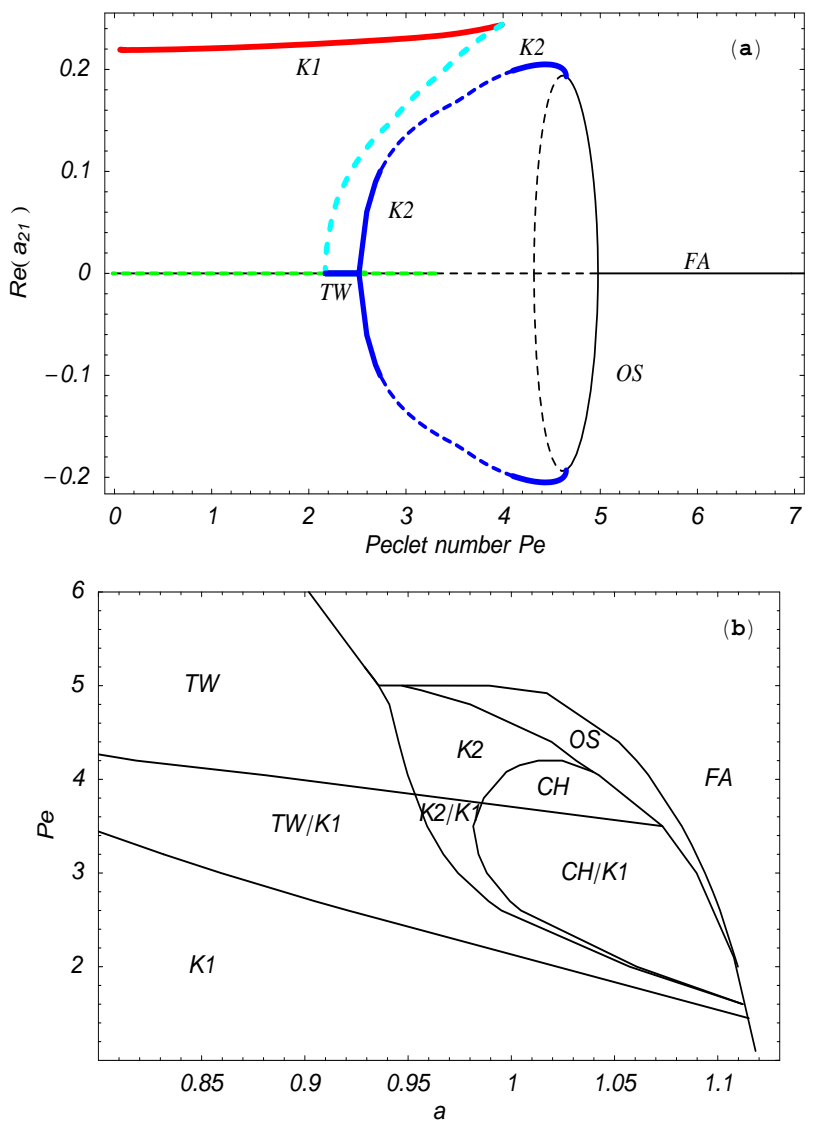

Figure 3: The top figure is the bifurcation diagram for the $N=5.2$ slice of Figure 2, corresponding to aspect ratio parameter $a=1$, i.e., infinitely thin rods. The lower figure is a virtual phase diagram of all stable states of the Doi-Hess kinetic theory for fixed nematic concentration $N=5.2$, variable shear rate $P e$ and variable molecular aspect ratio parameter $a$. The $a=1$ slice of the lower figure thus corresponds to the top figure, which establishes parameter contact with Figure 2 in our numerical continuation framework. 


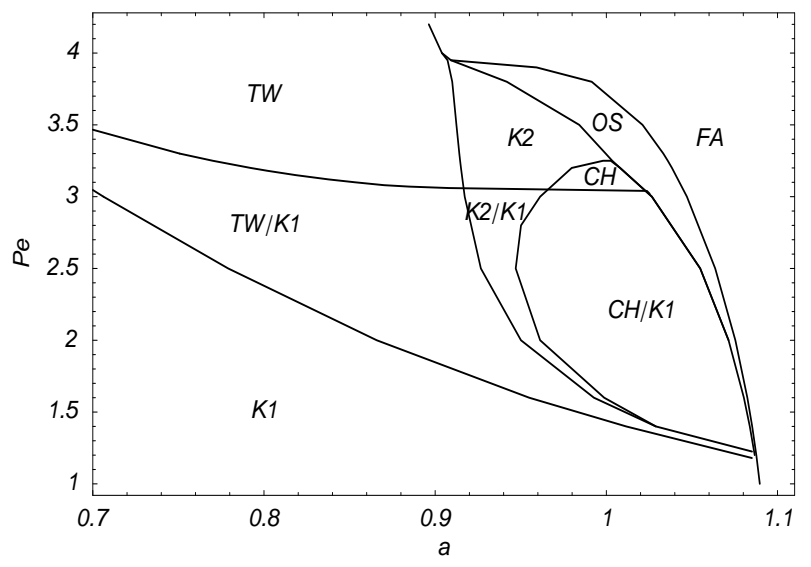

Figure 4: Phase diagram of all sheared monodomain attractors of the Doi-Hess kinetic theory for fixed nematic concentration $N=5$. The vertical axis is the normalized shear rate $P e$; the horizontal axis is the molecular aspect ratio parameter $a$. 

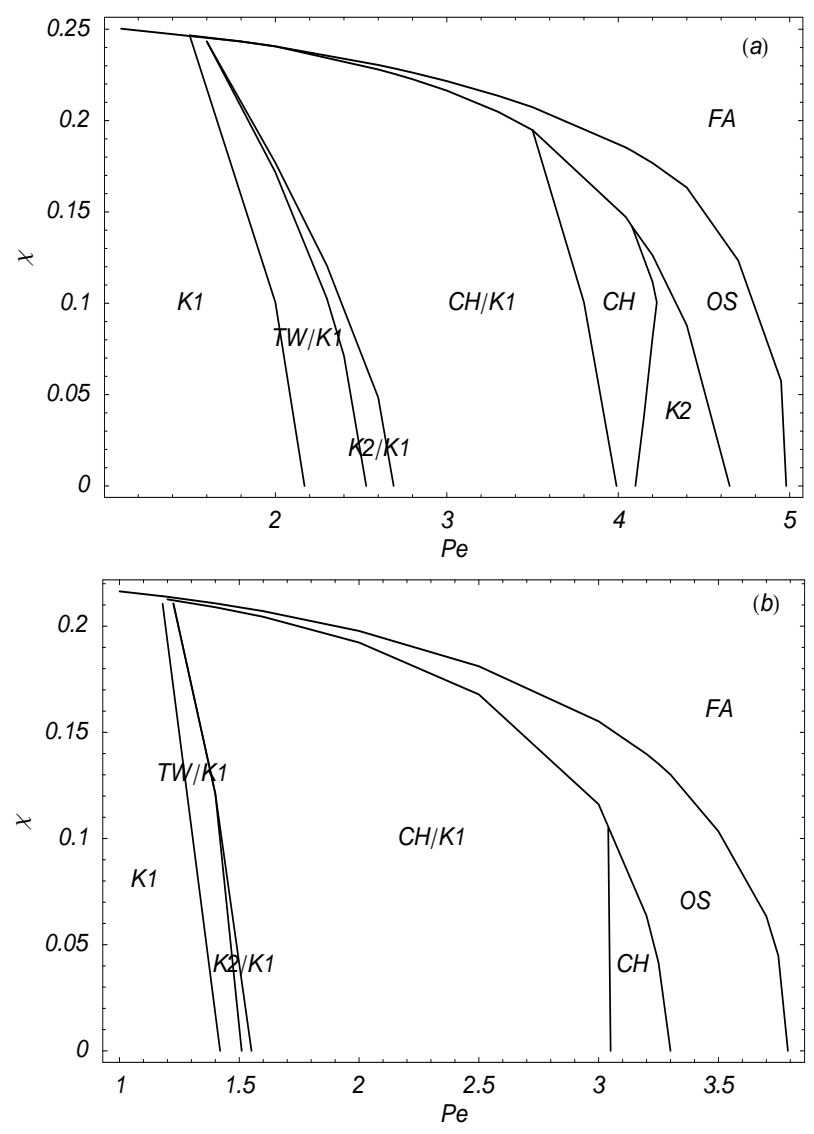

Figure 5: Phase diagrams for monodomain attractors of Doi-Hess kinetic theory for infinitely thin rods $(a=1)$ at two distinct nematic concentrations, $N=5.2$ (top) and $N=5$ (bottom). The horizontal axis is normalized shear rate $(P e)$, and the vertical axis $\chi$ is the ratio of extension rate $(\dot{\epsilon})$ to shear rate $(\dot{\gamma})$, see formula $(6)$. 


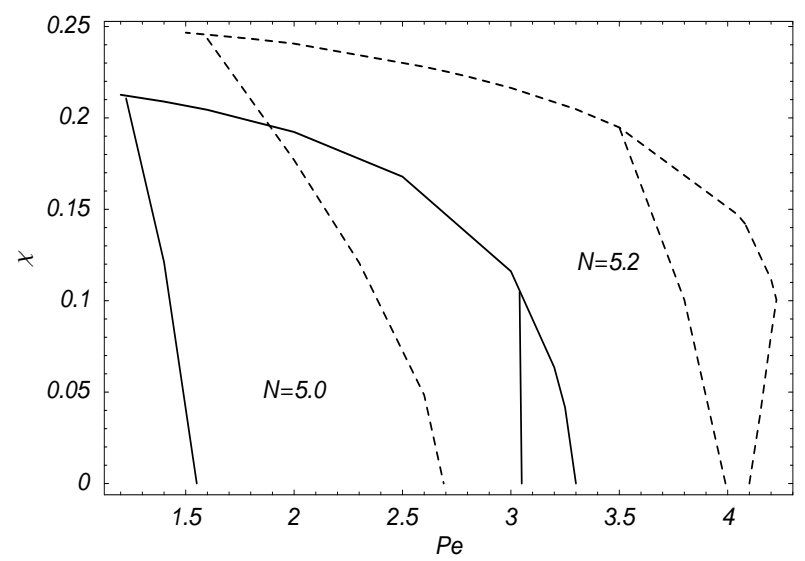

Figure 6: Comparison of chaotic regions in the parameter space of normalized shear rate $(P e)$ and extension rate $(\chi)$, for fixed nematic concentrations, $N=5$ (solid curves) and $N=5.2$ (dashed curves), and extremely thin rods $(a=1)$. 